\title{
Tratamiento con infliximab (anti-TNF alfa) en pacientes con enfermedad de Still del adulto. Experiencia de dos casos
}

\author{
Mª G. BONILLA HERNÁN, T. COBO IBÁÑEZ, E. DE MIGUEL MENDIETA, \\ E. MARTÍN-MOLA
}

Servicio de Reumatología. Hospital Universitario La Paz. Madrid

INFLIXIMAB (ANTI-TNF ALFA) IN THE TREATMENT OF ADULT STILL'S DISEASE. TWO CASES

\section{RESUMEN}

La enfermedad de Still del adulto es una enfermedad inflamatoria crónica de etiología desconocida. Los antinflamatorios no esteroideos (AINEs) y los glucocorticoides son la base del tratamiento, pero en casos refractarios o cuando la dosis de esteroides es muy elevada es necesario recurrir al tratamiento con fármacos antireumáticos modificadores de la enfermedad (FAME). Ultimamente se ha comenzado a utilizar tratamientos biológicos como alternativa terapéutica a los casos de enfermedad de Still del adulto refractaria al tratamiento convencional.

Presentamos nuestra experiencia satisfactoria del tratamiento con infliximab en dos pacientes con esta enfermedad.

PALABRAS CLAVE: Enfermedad de Still del adulto. Infliximab. Tratamiento con anti-TNF- $\alpha$.

\begin{abstract}
Adult Still's disease is a systemic inflammatory disorder of unknown etiolgy. First-line treatment for Still's disease includes nonsteroidal antiinflammatory drugs and corticosteroids. In refractory cases o when the dose of corticosteroid is unacceptbly high, other disease modifiying antirheumatic drugs have been used. But recent study showed the efficacy anti-TNF therapy in adult Sill's disease refractory to conventional therapy

We report a favourable response to infliximab in two patients who has proved resistant to conventional therapy
\end{abstract}

KEY WORDS: Adult Still's disease. Infliximab. Anti-TNF- $\alpha$ treatment.

Bonilla Hernán MG, Cobo Ibáñez T, De Miguel Mendieta E, Martín-Mola E. Tratamiento con infliximab (anti-TNF alfa) en pacientes con enfermedad de Still del adulto. Experiencia de dos casos. An Med Interna (Madrid) 2004; 21: 23-26.

\section{INTRODUCCIÓN}

La enfermedad de Still del adulto es una enfermedad inflamatoria crónica de etiología desconocida cuya prevalencia es de 1 por 100.000 habitantes y la incidencia es $0.16-0.34$ pacientes por 100.000 habitantes mayores de 16 años (1). Se caracteriza por fiebre, rash cutáneo, artralgias, artritis, leucocitosis y neutrofilia. Las manifestaciones sistémicas incluyen, serositis (pleuritis, pericarditis), dolor faríngeo, alteraciones en la función hepática, esplenomegalia y adenopatías. Dada su baja prevalencia carecemos de estudios terapéuticos controlados. Los AINE y los corticoides son la base del tratamiento precisando en ocasiones dosis por encima de $15-20 \mathrm{mg} / \mathrm{día}$ o incluso pulsos intravenosos de metilprednisolona. Para disminuir la dosis de esteroides o controlar las manifestaciones sistémicas en pacientes refractarios al tratamiento de primera línea se han usado distintos fármacos antireumáticos modifi- cadores de la enfermedad. En la actualidad el más utilizado es el metotrexate que ha demostrado ser útil y seguro en el tratamiento de las manifestaciones articulares y sistémicas y para reducir la dosis concomitante de corticoides (2), otros fármacos utilizados son ciclosporina, ciclofosfamida, sales de oro, antipalúdicos y azatioprina. Ultimamente,se han publicado algunos casos de enfermedad de Still del adulto refractaria a tratamientos convencionales con buena respuesta a terapias biológicas anti- TNF- $\alpha$.

\section{CASOS APORTADOS}

Caso 1: Mujer de 36 años de edad de nacionalidad peruana sin antecedentes personales de interés excepto una mola hidatiforme y dos abortos, que consulta por cuadro de fiebre de predominio vespertino de $39^{\circ} \mathrm{C}$, artromialgias, dolor abdominal tipo cólico y exantema.

Trabajo aceptado: 31 de julio de 2003

Correspondencia: Mª Gema Bonilla Hernán. Servicio de Reumatología. Hospital La Paz. Paseo de la Castellana, 261. 28046 Madrid. e-mail: magebo@eresmas.com 
A la exploración física general se objetivó temperatura de $38,6^{\circ} \mathrm{C}$, dos adenopatías laterocervicales izquierdas de $1 \mathrm{~cm}$ de tamaño y lesiones cutáneas en cuero cabelludo, frente, cara, cuello, región sacra, glútea y espalda, siendo el resto de la exploración general normal. En la exploración del aparato locomotor se apreció tumefacción en muñecas, rodillas y tobillos, limitación dolorosa a la flexión de la muñeca izquierda y disminución de fuerza en miembros inferiores. Ante los hallazgos clínicos se realizaron una serie de pruebas complementarias. En la analítica se objetivó leucocitosis de 15700 células con desviación izquierda ( $89 \%$ neutrófilos), 800 eosinófilos, anemia normocítica y normocrómica con hemoglobina de $10 \mathrm{mg} / \mathrm{dl}$, 508.000 plaquetas y VSG $125 \mathrm{~mm} / \mathrm{h}$. En el metabolismo del hierro se detectó hierro de $20 \mathrm{microgramos} / \mathrm{ml}$, ferritina: $3.157 \mathrm{ng} / \mathrm{ml}$, Transferrina: $300 \mathrm{mg} / 100 \mathrm{ml}$ con un índice de saturación de transferrina del 7\%. En la bioquímica GPT:45 U/ml, GGT:44U/ml, LDH: 725 U/L y FA: 359 U/L siendo el resto normal. El proteinograma mostraba un descenso de los niveles de albúmina y un aumento a 2 globulina. El estudio inmunológico fue negativo para FR, ANA, ANCA y los niveles de $\mathrm{C} 3$ y $\mathrm{C} 4$ fueron normales. El estudio microbiológico que incluía, mantoux, hemocultivos, serología, cultivo de parásitos en heces fue negativo excepto serología positiva Ig G para citomegalovirus. Entre las pruebas de imagen realizadas se incluyó radiografía de tórax, cráneo, manos, pies y pelvis encontrándose solamente un islote óseo coxal derecho siendo el resto normal. La ecografía abdominal y el TAC abdominopélvico fueron normales. Para completar el estudio se realizó PAAF de ganglio axilar sin alteraciones significativas y un EMG que objetivaba una miopatía leve-moderada de predominio en miembros superiores. Con todos estos datos la paciente fue diagnósticada de Enfermedad de Still del Adulto, ya que cumplía los criterios de Cush et al de 1987 y los de Yamaguchi et al (3). Se inició tratamiento con antinflamatorios (AINE), glucocorticoides a altas dosis (30 mg/día) y metotrexate inicialmente oral con mala respuesta terapéutica precisando ingreso hospitalario por lo que se decidió aumentar la dosis a $25 \mathrm{mg}$ intramuscular/semana a pesar de lo cual persistió la actividad clínica con marcada limitación funcional. Dada la mala respuesta con la terapia empleada se inició tratamiento biológico con infliximab en junio de 2001 a dosis de $5 \mathrm{mg} / \mathrm{kg}$ siguiendo la pauta establecida para los pacientes diagnosticados de Artritis Reumatoide a las semanas $0,2,6$ y cada 8 semanas posteriormente. Previa a todas las infusiones se efectuó exploración general, exploración de aparato locomotor, valoración del dolor con una escala analógica visual (EVA) de 0-100 mm, valoración de la actividad de la enfermedad con EVA de 0-100mm, rigidez matutina en minutos, número de articulaciones dolorosas (NAD) y tumefactas(NAT), DAS 28 y ana- lítica con hemograma, bioquímica con función renal y hepática, PCR, VSG, FR, ANA y metabolismo de hierro, cuyos resultados aparecen reflejados en la Tabla I. Inicialmente se continuó con mismo tratamiento metotrexate $25 \mathrm{mg} / \mathrm{semana}$, ácido fólico, indometacina $75 \mathrm{mg} /$ día, prednisona $30 \mathrm{mg} /$ día, calcio y vitamina D. A lo largo del seguimiento la paciente evolucionó satisfactoriamente con disminución de todos los parámetros de actividad tanto clínica, como de laboratorio. (Tabla I)..La mejoría ha permitido disminuir de forma progresiva la dosis de esteroides y de AINE. En la actualidad el tratamiento es de $10 \mathrm{mg} /$ día de prednisona y $25 \mathrm{mg}$ de indometacina, manteniéndose la misma dosis de metotrexate. El tratamiento es bien tolerado y no ha presentado efectos secundarios. Se han realizado titulación de anticuerpos antinucleares y anti-dnas en todas las visitas. Tras la tercera dosis el título de ANA fue 1/80 homogéneo, tras la cuarta $1 / 40$ moteado y tras la quinta se negativizaron, los antiDNA siempre han sido negativos.

Caso 2: Mujer de 43 años de edad. Entre sus antecedentes personales solamente refería amigdalectomía. La paciente ingresó en el servicio de reumatología por cuadro de fiebre, artralgias, artritis y rash cutáneo. En la exploración física destacaba discreta palidez cutáneo mucosa, hepatomegalia de 1-2 traveses de dedos. Con dolor en articulación acromioclavicular izquierda, muñecas, rodillas y metatarsofalangicas, junto con tumefacción en hombros y muñecas. En la analítica se detectó leucocitosis de 22.300 células con desviación izquierda (90\% neutrofilos), VSG de 109, y aumento de transaminasas (GOT:64 U/L, GPT:80 U/L, GGT;43 U/L),ferritina de 241 $\mathrm{ng} / \mathrm{ml}$. El proteinograma presentaba una disminución de albúmina y un aumento de alfa.2 y gammgaglobulina. La serología para RPR, VHC, VHB, VIH, fue negativa al igual que un frotis faríngeo, una biopsia cutánea y los hemocultivos realizados. El FR y losANA fueron negativos y el complemento normal. Las Rx de tórax, manos, pies, rodillas, eco abdominal y ecocardiograma fueron también normales. El Mantoux fue positivo. Con estos síntomas se diagnosticó de enfermedad de Still del adulto. Inicialmente recibió tratamiento con AINES, esteroides a alta dosis y profilaxis con isoniazida 300 $\mathrm{mg} / \mathrm{día}$ por mantoux positivo. Posteriormente dada la persistencia de la clínica se inició tratamiento con sales de oro intramuscular que tuvo que suspender por proteinuria, iniciándose tratamiento con metotrexate a dosis ascendente hasta $25 \mathrm{mg} / \mathrm{semana}$ intramuscular. A pesar de este tratamiento y de infiltraciones locales con esteroides de liberación retardada, la paciente continuó con actividad clínica importante y oligoartritis con marcada sinovitis e incapacidad funcional en carpo derecho. $\mathrm{Al}$ igual que en la paciente anterior y ante la falta de respuesta a los tratamientos empleados se planteó el inicio de

TABLA I

EVOLUCIÓN CLÍNICA Y ANALÍTICA DE LA PRIMERA PACIENTE

\begin{tabular}{lccccccccc}
\hline Visita & Ferritina & $H B$ & NAD & NAT & VGP & VSG & DAS & $P C R$ & $H A Q$ \\
\hline $21 / 06 / 01$ & - & 9,4 & 13 & 13 & 53 & 88 & 6,91 & 13,7 & 1,5 \\
$05 / 07 / 01$ & - & 8,4 & 7 & 22 & 73 & 82 & 6,92 & 10,5 & - \\
$02 / 08 / 01$ & 82 & 9,1 & 18 & 22 & 25 & 53 & 6,82 & 1,62 & - \\
$28 / 09 / 01$ & 71 & 9,5 & 9 & 9 & 23 & - & - & 7,2 & 1,12 \\
$23 / 11 / 01$ & 10 & 10,1 & 7 & 3 & - & 39 & - & 0,6 & 1 \\
$18 / 01 / 02$ & 13 & 10 & 3 & 4 & 17 & 30 & 4,15 & 0,6 & 0,75 \\
$15 / 03 / 02$ & 10 & 10,4 & 3 & 3 & 12 & 24 & 3,84 & - & - \\
$10 / 05 / 02$ & - & 10,8 & 0 & 1 & 8 & 28 & 2,73 & - & - \\
\hline
\end{tabular}


TABLA II

EVOLUCIÓN CLÍNICA Y ANALÍTICA DE LA SEGUNDA PACIENTE

\begin{tabular}{lccccccccc}
\hline Visita & Ferritina & $H B$ & NAD & NAT & VGP & VSG & DAS & $P C R$ & $H A Q$ \\
\hline $06 / 06 / 02$ & 76 & 12,9 & 3 & 2 & 98 & 31 & 5,14 & 71,5 & 1,75 \\
$20 / 06 / 02$ & & 11,7 & 3 & 1 & 35 & 27 & 3,64 & 43,5 & 0,75 \\
$18 / 07 / 02$ & 82 & 12,6 & 2 & 3 & 42 & 23 & 4,15 & 38,5 & 0,87 \\
\hline
\end{tabular}

tratamiento con infliximab como uso compasivo. Se administraron tres dosis de tratamiento intravenoso $(3 \mathrm{mg} / \mathrm{kg})$ a la 0,2 y 6 semanas. El tratamiento fue bien tolerado y durante el seguimiento los ANA fueron negativos. Antes de la cuarta dosis presentó infección de un lipoma localizado en la región mamaria derecha próxima a la línea anterointerna axilar que precisó tratamiento antibiótico y posterior extirpación con resolución del cuadro infeccioso. En el momento actual la paciente se encuentra con dosis bajas de esteroides y metotrexate intramuscular y ha reiniciado tratamiento con infliximab. Durante las seis semanas de tratamiento no presentó síntomas sistémicos y disminuyó de forma importante la intensidad de dolor (Tabla II).

\section{DISCUSIÓN}

En la enfermedad de Still del Adulto se han detectado elevaciones de las concentraciones de factor de necrosis tumoral alfa (TNF- $\alpha$ ), interleucina 6 (IL-6) e interferón gamma (4). Además, se ha observado que la inhibición de TNF- $\alpha$ con agentes antagonistas como la talidomida produce mejoría clínica (5). Recientemente también se ha demostrado que la citoquina IL18 esta elevada en el suero de los pacientes diagnosticados de enfermedad de Still del adulto durante la fase aguda, (6) y se ha visto que el TNF induce la expresión de IL 18 en el tejido sinovial (7), por lo que se piensa que los anti-TNF pueden reducir los niveles de IL 18 en el suero de estos pacientes, de hecho se ha demostrado que el infliximab reduce los niveles de IL 18 en pacientes con artritis reumatoide (8). Esto junto que se haya demostrado una rápida reducción de las concentraciones séricas de IL-6 en los pacientes tratados con infliximab, ha hecho que se plantee el uso de terapias biológicas que actúan neutralizando la acción de la citocina TNF- $\alpha$ como el infliximab (anticuerpo monoclonal quimérico contra el factor necrosis tumoral y el embrel (receptor soluble tipo II del TNF- $\alpha$ ) como una alternativa al tratamiento de los pacientes con ésta enfermedad y actividad persistente con el tratamiento convencional. Esto, unido al hecho de algunos casos publicados en la literatura nos animó a la utilización de forma compasiva de este fármaco. Nuestros resultados confirman la experiencia recogida en la literatura hasta el momento, sobre el tratamiento con infliximab en pacientes con enfermedad de Still del adulto (9-11).

Las tres series publicadas de tratamiento con infliximab incluyen un número reducido de pacientes, 2, 3 y 6 respectivamente. En la primera dos pacientes con enfermedad de Still de adulto agresiva recibieron tratamiento con $3 \mathrm{~m} / \mathrm{kg}$ de peso en las semanas $0,2,6$ y después cada 8 semanas, objetivándose una mejoría clínica desde las primera dosis de tratamiento (9). En la segunda serie con 3 pacientes diagnosticados de enfermedad de Still del adulto según los criterios de
Yamagushi que recibieron tratamiento con infliximab con dosis de $3 \mathrm{mg} / \mathrm{kg}$ de peso a las semanas $0,2,6$ y cada 8 semanas posteriormente, uno presentó una reacción urticariforme difusa severa que obligó a suspender tratamiento y los otros presentaron mejoría clínica y articular inicial, con empeoramiento en las semanas 20 y 28 respectivamente, probablemente secundario a un excesivo tiempo entre las infusiones por lo que se redujo el intervalo a 4 semanas con la reaparición de la mejoría clínica (10). En la tercera los pacientes fueron diagnosticados de Enfermedad de Still del adulto según los criterios de Yamaguchi y recibieron tratamiento con infliximab a dosis de $3-5 \mathrm{mg} / \mathrm{kg}$ de peso según la gravedad de la sintomatología a las semanas $0,2,6$ y después cada 6-8 semanas. Tan solo uno tuvo una reacción durante la infusión que cedió con la interrupción del tratamiento durante 1 hora sin presentar problemas posteriormente y todos los pacientes mejoraron con el tratamiento (11).También hay experiencia de tratamiento con etanercept en pacientes con enfermedad de Still del adulto. Hay 2 series $(12,13)$,y un caso publicado. La primera incluye 12 pacientes con Enfermedad de Still del adulto, de los cuales tres presentaban fiebre y exantema. Todos recibieron tratamiento con etanercept $25 \mathrm{mg}$ subcutáneo 2 veces por semana. Se objetivó mejoría ACR $50 \%$ en 5 pacientes y ACR 70\% en dos. En dos de los tres pacientes con fiebre y exantema cutáneo se suspendió por persistencia de los síntomas, en el tercero desapareció la fiebre y el rash pero no presentó mejoría de la clínica articular. En la segunda 5 pacientes diagnosticados de enfermedad de Still del adulto también según los criterios de Yamaguchi con afectación articular y sistémica, recibieron tratamiento con $25 \mathrm{mg}$ de etanercept 2 veces a la semana. En ellos se objetivó mejoría a las 3 semanas y como efectos secundarios destacan la muerte súbita de uno de ellos a la sexta semana y una celulitis. Hay un caso publicado de una mujer de 35 años diagnosticada de enfermedad de Still del adulto con curso agresivo que incluía amiloidosis cardiaca. La paciente tenía persistencia de la actividad clínica a pesar de los múltiples tratamientos empleados que incluían alta dosis de esteroides, pulsos intravenosos de esteroides, bolos de ciclofosfamida, metotrexate, cloroquina, d-penicilamina e incluso plasmaferesis. Presentó mala respuesta con los múltiples tratamientos empleados y complicaciones secundarias graves derivada de los efectos secundarios de estos tratamientos. Iniciándose tratamiento con etanercept con una respuesta favorable a dicho tratamiento (14).

Aunque en el momento actual es poca la experiencia en el tratamiento de enfermedad de Still del adulto con terapias biológicas anti TNF-a, nuestros resultados junto con la experiencia reunida de la literatura hacen pensar que el infliximab puede ser una alternativa terapéutica en pacientes diagnosticados 
de enfermedad de Still del adulto refractarios al tratamiento convencional. Aunque, sin duda es cierto que son necesarios estudios multicéntricos rabdomizados controlados con un mayor número de pacientes para definir mejor el efecto y la seguridad de estos tratamientos en este grupo de pacientes y para valorar la dosis eficaz y el intervalo entre dosis.

\section{Bibliografía}

1. Montilla CA, Medina JA, Conde E. Enfermedad de Still del adulto Seminarios de la Fundación Española de Reumatología 2001; 2; 335343.

2. Fautrel B, Borget C, Rozenberg S. J Rheumatol 1999; 26: 373-378.

3. Yamaguchi M, Ohta A, Tsunematsu, Kasukawa R, Mizushima Y, Kashiwagi H et al. Preliminary criteria for classification of adult Still's disease. J Rheumatol 1992;19: 424-430.

4. Hoshino T, Ohta A, Yang D. Elevated serum Interleukin-6, Interferon g and tumor necrosis factor a levels in patients with adult Still's disease. J Rheumatol 1998; 25: 396-398.

5. Stambe C, Wicks IP. TNF-alpha and response of treatment-resistant adult-onset Still's disease to thalidomide. Lancet 1998: 352: 544-5.

6. Kawashima M, Yamamura M, Taniai M, Yamauchi H, Tanimoto T, Kurimoto M, et al. Level of interleukin-18 and its binding inhibitors in the bloo circulation of patients with adult-onset Still's disease. Athritis Rheum 2001; 44: 550-560.

7. Gracie JA, Forsey RJ, Chan WL, Gilmour A, Leung BP, Greer MR et al. A proinflammatory role for IL-18 in rheumatoid arthritis. J Clin Invest 1999; 104: 1393-1401.

8. Pittoni V, Bombardieri M, Spinelli FR, Scrivo R, Alessandri C, Conti

Spadaro A, Valesini G. Anti-tumour necrosis factor (TMF) alpha rheumatoid arthritis(infliximab) selectively down regulates the production interleukin (IL) 18 but not of IL12 an IL 13. Ann Rheum Dis 2002; 61: 723-725.

9. Aurrecochea E, González-Suárez, Martínez-Taboada VM, Sánchez-Andrada S, Peña JL, Blanco R, Rodríguez-Valverde V. Infliximab en la enfermedad de Still del adulto: dos casos. Rev Esp Reumatol 2001; 28: 217

10. Cavagna L, Caporali R, Epis O, Bobbio-Pallavicini F, Montecucco C. Infliximab in the treatment of adult Still's disease refractory to conventional therapy. Clin Exp Rheumatol 2001; 19: 329-332.

11. Kraetsch HG, Antoni C, Kalden JR, Manger Erlangen B. Succesful treatment of a small cohort of patients with adult onset of Still disease with infliximab. Arthritis Rheum 2001; 44: 395.

12. Elaine Husni M, Maier AL, Mease PL, Overman SS, Fraser P, Gravalles EM, Weinblatt ME. Etanercept in the treatment of adult patients with Still's disease. Arthritis Rheum 2002; 48: 1171-1176.

13. Tamesis ER, Reginato AM, Hubsscher O. In recalcitrant Adult Onset Still's. Dis Rheum 2000; 43: S229.

14. Asherson RA, Pascoe L. Adult onset Still's disease: response to Enbrel. Ann Rheum Dis 2002; 61: 859-860. 